\title{
Actividade física habitual em crianças. Diferenças entre rapazes e raparigas.
}

V. P. Lopes

\section{A. M. Monteiro \\ T. Barbosa \\ P. M. Magalhães}

Instituto Politécnico de Bragança

Portugal

https://doi.org/10.5628/rpcd.01.03.53

\section{RESUMO}

O objectivo central deste estudo foi caracterizar o perfil da actividade física de crianças de ambos os sexos, de uma escola da cidade de Bragança, ao longo de 4 dias. A amostra foi constituída por 14 raparigas e 11 rapazes de $9,5 \pm 0,6$ anos de idade. $A$ actividade física foi avaliada através do monitor de actividade física CSA 7164, ao longo de 4 dias consecutivos, dois dias de semana - Segunda e Sexta, e dois dias de fim de semana Sábado e Domingo. O CSA foi colocado no punho do braço não dominante. Após a colecta dos dados foi calculada a média de contagens por minuto em cada dia e em cada hora. A MANOVA de medidas repetidas foi utilizada para analisar as diferenças entre sexos, entre os dias e entre as horas em cada dia na intensidade de actividade física. O coeficiente de correlação intraclasse (R) foi utilizado para analisar a variabilidade intraindividual na actividade física habitual. Os resultados indicam diferenças significativas entre a intensidade de actividade física dos dias de semana e o fim de semana. Não se verificaram diferenças significativas entre os rapazes e as raparigas. Ocorreu uma grande variabilidade intraindividual na actividade física habitual, especialmente no fim de semana.

Palavras-chave: actividade física, crianças, variabilidade intraindividual, diferenças entre sexos.
J. A. R. Maia

Faculdade de Ciências do Desporto e de Educação Física Universidade do Porto Portugal
Daily Physical Activity in Children. Gender Differences.

The purpose of this study was to identify the physical activity pattern of boys and girls during 4 days. The sample comprises 14 girls and 11 boys of 9,5 0,6 years of age. Physical activity was assessed with Computer Science and Applications (CSA - 7164) activity monitor, during 4 days - two week-days (Monday and Friday), and during the weekend (Saturday and Sunday). The activity monitor was worn on the wrist of the non-dominant arm. After the data collection we calculated the mean of activity per minute in each day and in each hour. The repeated measures MANOVA were used to analyse the changes between each day's activity and between hours in each day, and gender differences. Intraclass correlation coefficient $(R)$ was used to analyse the intraindividual variability of physical activity. Results indicated a significant main effect of days, meaning that the pattern of activity changed over days. The differences occurred between Friday and Saturday, and between Saturday and Sunday. At the weekend less physical activity was registered than in weekdays in both boys and girls. There was no significant main effect for gender, meaning that the physical activity of boys and girls are similar. There was also no interaction between gender and days, meaning that the change in activity pattern was similar for both boys and girls. We found an enormous intraindividual variability, special during the weekend days.

Keywords: physical activity, children, intraindividual variability, gender differences. 


\section{INTRODUÇÃO}

É consensual a noção de que a actividade física habitual é um comportamento de grande importância para a promoção de um estilo de vida saudável, tanto na infância e juventude como na idade adulta. Nos adultos, a actividade física regular tem uma influência benéfica sobre alguns factores relacionados com a saúde, estando associada ao aumento da longevidade $(9.26,7)$, a um decréscimo do risco de doenças coronárias $(6,26,7)$, na diminuição de alguns dos factores de risco que lhes estão associados, como a obesidade e o stress emocional, e uma acção profiláctica em patologias degenerativas do sistema osteo-articular $(11,7)$. Nas crianças, a actividade física está associada à redução de factores de risco de doenças cardiovasculares: pressão sanguínea (26), presença de lipoproteínas de baixa densidade (13, 26) e obesidade (10). Estas evidências levaram ao reconhecimento, por parte de organizações insuspeitas e de grande credibilidade - por ex. American Heart Association (14), de que a inactividade física é um dos maiores factores de risco para a contracção de doenças cardiovasculares, tendo como consequência o desenvolvimento de objectivos, por parte de departamentos governamentais de alguns países - por ex. U.S Department of Health and Human Services (31), para a promoção da actividade física regular e o delineamento de orientações específicas de exercitação para a população adulta (23).

A infância e a juventude são consideradas idades determinantes no ganho de hábitos duradoiros de actividade física até à idade adulta. Parece ser razoável assumir que as crianças que sejam fisicamente activas sejam aquelas que venham a manter esse hábito enquanto adultos. De facto, a promoção da actividade física na infância e juventude baseia-se, em parte, no pressuposto de que os hábitos de actividade física se desenvolvem durante estes períodos e se mantêm até à idade adulta. É patente que as crianças apreciam actividades de natureza lúdico-motora. Dando-lhes oportunidade, a generalidade das crianças opta por jogos e actividades motoras de cariz diversificado. As crianças são, de facto, por natureza fisicamente activas. Existem, no entanto, poucos dados sobre o padrão (frequência, duração, intensidade, tipo, gasto energético) de actividade física das crianças, uma vez que as investigações se têm centrado sobretudo noutros escalões, essencialmente em adolescentes e adultos. Além disso, o facto de os diferentes estudos usarem procedimentos distintos na avaliação da actividade física (inquéritos, observação, monitores de actividade física, monitores da frequência cardíaca) dificulta a comparação dos resultados, não se conhecendo qualquer estudo de meta-análise que coloque alguma ordem na disponibilidade de informação em crianças até aos 10 anos de idade.

A esta falta de dados não é com certeza alheia a dificuldade acrescida de avaliação da actividade física neste escalão etário. Existem diversos meios para avaliação da actividade física habitual. O inquérito é um dos instrumentos mais utilizados em estudos de carácter epidemiológico, no entanto, o seu grau de precisão é baixo. Esta imprecisão da medida é agravada quando se trata de avaliar crianças, dado que possuem um grau muito baixo de compreensão das questões e um nível de memória que não lhes permite recordar, de forma adequada e precisa, as suas actividades diárias. Com o questionário torna-se difícil captar todo o tipo de actividade física. De facto, se o questionário contém questões do tipo fechado, a precisão está também dependente da forma como estas registam todas as actividades físicas (15). Nas crianças este problema é agravado pelo facto da actividade física ser altamente transitória, caracterizada por períodos curtos e intermitentes de actividade física intensa com períodos frequentes de repouso (4). Para eliminar muitos destes problemas torna-se necessário recorrer a métodos alternativos que não dependam dos sujeitos a avaliar e, por conseguinte, não exijam das crianças a capacidade para recordarem as suas actividades. Os detectores mecânicos e electrónicos do movimento (por ex. pedómetros e acelerómetros), que são extremamente práticos e fiáveis, eliminam muitos destes problemas.

$\mathrm{O}$ interesse em avaliar a actividade física em qualquer população baseia-se na necessidade de estabelecer o estado corrente da actividade física dessa população e determinar se a população se encontra dentro dos critérios apropriados e indispensáveis a um óptimo estado de saúde. O Council for Physical Education do National Association for Sport and Physical Education (12) dos EUA recomenda que as crianças deverão acumular pelo menos 30 a 60 min de actividade físi- 
ca apropriada à idade e ao estado de desenvolvimento, em todos, ou na maior parte, dos dias da semana. Simons-Morton et. al (26) verificaram, em crianças de 8/9 anos de idade de ambos os sexos, que a média diária de períodos em actividades físicas moderadas e vigorosas com duração superior a 10 minutos foi de 1,7. A ocorrência de episódios de actividades físicas moderadas e vigorosas foi significativamente superior fora da escola. Os autores referem que, embora a maioria das crianças tivessem referido ter alguma actividade por dia, uma proporção substancial de crianças indicou menos de uma actividade física moderada e vigorosa diária com duração igual ou superior a 10 minutos, o que sugere que muitas crianças podem não ter uma quantidade de actividade física adequada. Sleap e Warbuston (27) estudaram 56 crianças de 5 a 11 anos de idade de 4 regiões de Inglaterra ao longo de 10 meses. As observações foram efectuadas durante os intervalos lectivos, os períodos de refeições, as aulas de Educação Física e durante o tempo livre fora da escola. As crianças passaram $34,4 \%$ do tempo de observação em actividades físicas moderadas e vigorosas. Foram mais activas durante os intervalos lectivos do que durante o tempo livre passado fora da escola. Apenas em $14 \%$ das crianças observadas foram registados períodos continuados de 20 minutos ou mais de actividade física. Magalhães (18) estudou a actividade física de 120 crianças durante o recreio escolar através do acelerómetro Tritrac-R3D e verificou que as crianças apresentam um predomínio de actividade física de intensidade baixa, não realizando no mínimo 30 minutos de actividade física moderada a vigorosa.

Parece haver a ideia de que os rapazes são mais activos do que as raparigas. No entanto, os resultados das investigações são contraditórios. Por exemplo, Welsman e Armstrong (33) verificaram que os rapazes passavam significativamente mais tempo em actividades físicas moderadas e intensas do que as raparigas e, no entanto, Atkins et al. (3) não encontram diferenças significativas entre rapazes e raparigas. Por outro lado, Santos (25), ao analisar a actividade habitual em 157 indivíduos de ambos os sexos com idades compreendidas entre os 8 e os 16 anos, apurou que os rapazes foram mais activos que as raparigas e que a actividade física tem um declínio acentuado ao longo da idade, particularmente nas raparigas. Também Magalhães (18) encontrou, tanto na avaliação por questionário, como na avaliação através de acelerómetro, índices superiores de actividade física no tempo de lazer, sobretudo quanto à actividade física de intensidade elevada.

Um aspecto importante relativo à caracterização do padrão de actividade física habitual é a variabilidade intraindividual, isto é, a diferença de actividade física de dia para dia. Para ter um impacto positivo na saúde a actividade física deve ser regular numa base diária. Trost et al. (29) indicam valores de R relativamente elevados (0,77; IC95\%: 0,73-0,82) quando analisaram a variabilidade intraindividual da actividade física ao longo de quatro dias.

O objectivo central deste estudo é caracterizar o perfil de actividade física, no que diz respeito à intensidade, de crianças de ambos os sexos, de uma escola da cidade de Bragança, ao longo de 4 dias - dois dias de semana e fim-de-semana, analisando as diferenças entre os diferentes dias e entre rapazes e raparigas.

\section{MATERIAL E MÉTODOS}

\subsection{Amostra}

A amostra foi constituída por 15 raparigas e 11 rapazes com 9,5 $\pm 0,6$ anos de idade de uma escola do $1^{\circ}$ Ciclo do Ensino Básico da cidade de Bragança.

\subsection{Avaliação da actividade física}

A actividade física foi avaliada através do monitor de actividade física CSA versão AM7164 (Computer Science and Aplications, Inc.). O monitor de actividade física CSA (5,1 X 4,1 X 1,5cm, 43g) é um acelerómetro uniaxial que mede a aceleração na direcção vertical. O monitor foi construído para detectar uma magnitude de aceleração entre 0,05 a 2,0 G, com uma frequência de resposta entre 0,25 e $2,5 \mathrm{~Hz}$. O CSA contém um microprocessador que digitaliza e filtra o sinal de aceleração, converte o sinal num valor numérico e acumula este valor como contagens de movimento ao longo de um intervalo que é determinado pelo investigador.

Cada criança foi monitorizada ao longo de 4 dias dois dias de semana (Segunda e Sexta) e dois dias de fim de semana (Sábado e Domingo). Quatro dias podem ser considerados como reflectindo de forma fiável a actividade física habitual (17). O monitor de 
actividade física foi colocado no punho do braço não dominante, sendo retirado apenas quando a criança ia tomar banho, já que não é resistente à água. $\mathrm{A}$ opção pela colocação no punho ao invés da anca, ficou a dever-se a circunstâncias operacionais, já que a generalidade das crianças não usava cinto, especialmente as raparigas. Melanson e Freedson (22) verificaram que as contagens estavam significativamente correlacionadas $(0,66 \leq \mathrm{r} \leq 0,81)$ com a estimação do dispêndio energético, independentemente do local de colocação do monitor (punho, anca ou tornozelo). A melhor equação de regressão para estimar o gasto energético foi aquela que usou as contagens obtidas com o monitor colocado no punho. Ao contrário destes resultados a melhor equação de regressão encontrada por Swartz et al. (28) usava as contagens com o acelerómetro CSA colocado na anca. As amostras destes estudos eram constituídas por sujeitos adultos. Não é do nosso conhecimento que este tipo de estudos existam com crianças. As contagens obtidas com o monitor colocado no punho são sistematicamente mais elevadas do que quando colocado na anca - no estudo de Swartz et al. (2000) a média das diferenças foi de 1741 contagens.

As contagens são posteriormente transferidas, através de uma interface apropriada, para um computador e tratadas para, posteriormente, serem analisadas.

\subsection{Procedimentos estatísticos}

Após a colecta dos dados foi calculada a média de contagens por minuto em cada hora e em cada dia. A MANOVA de medidas repetidas (dias X sexo) foi usada para analisar as diferenças entre rapazes e raparigas na mudança de actividade física ao longo dos 4 dias de observação.

Foi calculado o coeficiente de correlação intra-classe (R) para estimar a variação intraindividual ao longo dos 4 dias de observação.

A MANOVA de medidas repetidas (horas X sexo) foi também utilizada para analisar as diferenças de actividade física entre os sexos ao longo de cada dia. O cálculos foram realizados numa primeira fase na folha de cálculo Excel 2000 e posteriormente no SPSS 9.0.

\section{RESULTADOS}

No Quadro 1 são apresentadas as médias e os desvios-padrão das contagens de actividade física por minuto em cada dia de observação nos rapazes e nas raparigas.

Quadro 1 - Média e desvio padrão das contagens por minuto em cada dia de observação

\begin{tabular}{lcc} 
Dia & Raparigas & Rapazes \\
\hline Sexta & $1317,40 \pm 305,93$ & $1477,91 \pm 310,10$ \\
Sábado & $1126,10 \pm 318,78$ & $1273,94 \pm 304,47$ \\
Domingo & $1036,71 \pm 274,43$ & $974,68 \pm 243,64$ \\
Segunda & $1138,26 \pm 240,22$ & $1233,28 \pm 193,70$
\end{tabular}

Os resultados da MANOVA indicam um efeito estatisticamente significativo dos dias $[\Lambda=0.401 ; \mathrm{F}$ (3, 21) $=10.459 ; \mathrm{p}=0.000]$, o que revela que o padrão (intensidade) de actividade física se altera ao longo dos dias. As diferenças ocorrem entre a Sexta e o Sábado $[F(1,23)=5.247 ; p=0.031]$ e entre o Sábado e o Domingo $[\mathrm{F}(1,23)=24.743 ; \mathrm{p}=$ $0.000]$. Nos dias de fim de semana foram registados valores menores de actividade física do que nos dias de semana tanto nos rapazes como nas raparigas. Não se verificaram efeitos significativos do sexo e da interacção sexo $\mathrm{X}$ dias, o que significa que o padrão (intensidade) de actividade física dos rapazes e raparigas foi similar (Figura 1).

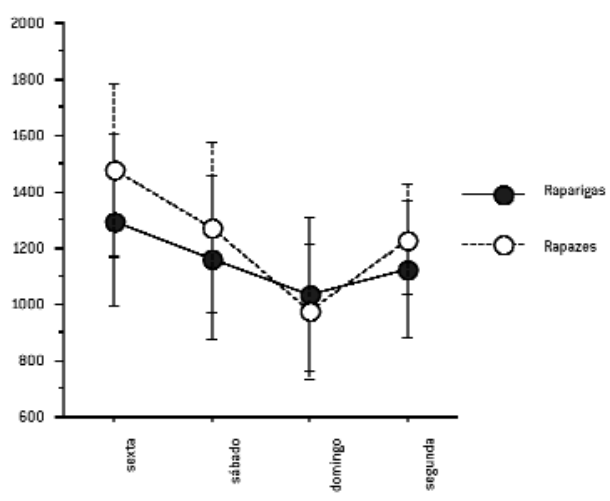

Figura 1 - Perfil das médias de contagens por minuto e por dia ao longo dos quatro dias de observação. 
Os resultados do coeficiente de correlação intra-classe (R) são apresentados no Quadro 2. Os valores são baixos com a excepção do valor encontrado para as raparigas nos dias de semana $(0,90)$.

Quadro 2 - Valores do coeficiente de correlação intra-classe $[R]$

\begin{tabular}{lcc} 
& Rapazes & Raparigas \\
\hline 4 dias & 0,51 & 0,77 \\
Dias da semana & 0,27 & 0,90 \\
Fim de semana & 0,39 & 0,04
\end{tabular}

Sexta

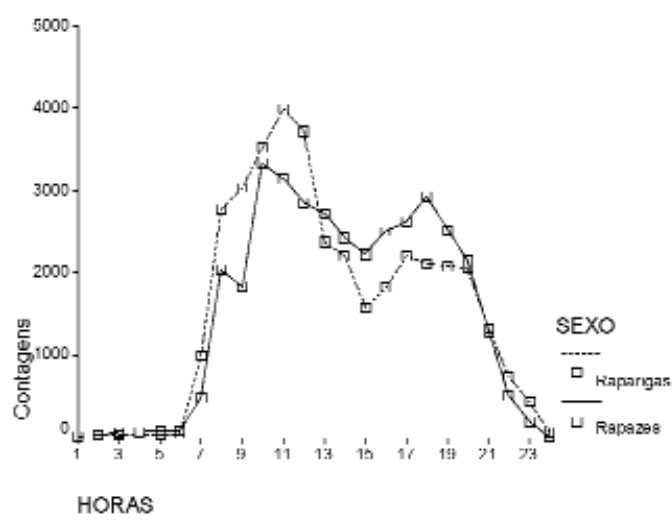

Domingo

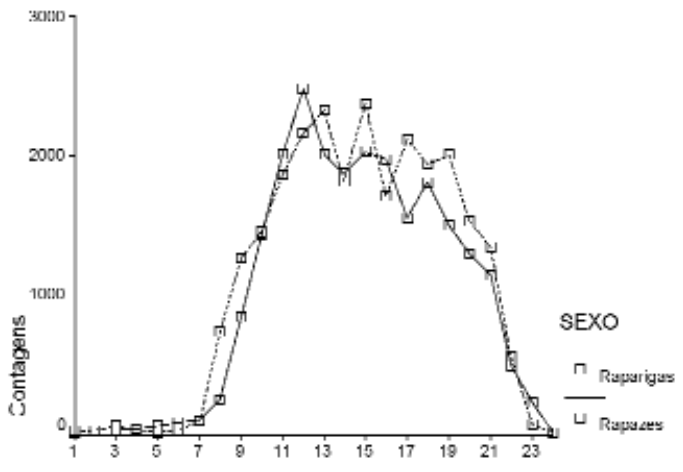

HORAS
A MANOVA de medidas repetidas não indica diferenças significativas entre os rapazes e as raparigas em qualquer dos quatro dias da semana. Também não indica interacção significativa entre o sexo e as horas do dia em qualquer dos quatro dias de observação. De facto, os gráficos da Figura 2 mostram claramente que o perfil de actividade física dos rapazes e raparigas é similar ao longo do dia nos diferentes dias da semana.

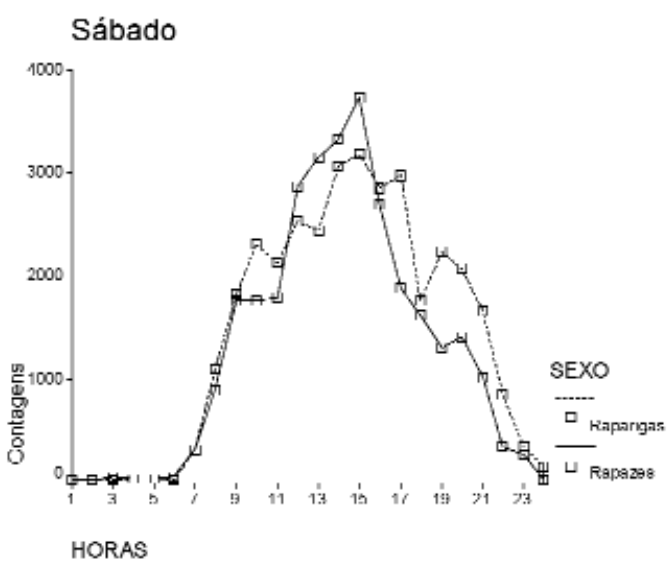

Segunda

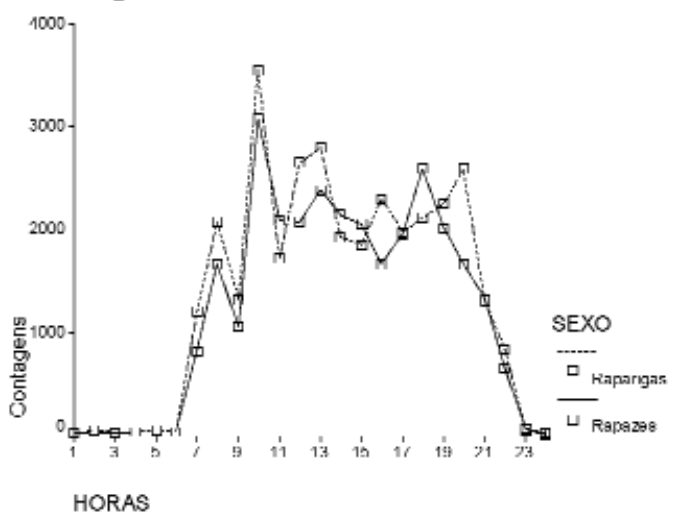

Figura 2 - Perfil das contagens por minuto em cada hora ao longo do dia nos quatro dias de observação 
Durante os dias de semana (Sexta e Segunda), o período de actividade mais intensa (10 horas) coincide com o intervalo/recreio escolar. O domingo apresenta os valores mais baixos de actividade física, tanto nos rapazes como nas raparigas, e um perfil mais suavizado ao longo do dia.

\section{DISCUSSÃO}

O presente estudo, usando uma metodologia de avaliação da actividade física tecnologicamente avançada - a acelerometria - pretendeu fazer uma caracterização da actividade física de crianças de ambos os sexos durante um período de quatro dias. O padrão de actividade física é geralmente descrito através da frequência, intensidade e duração, sendo, por vezes, também usado o dispêndio energético. No presente estudo estudou-se apenas a intensidade, representada pelo número ou quantidade de contagens. A duração e a frequência - que são geralmente apresentadas em conjunto como o número de períodos ou tempo total que a criança está activa em diferentes categorias de actividade (leve, moderada ou intensa) - não foram tidas em consideração, dado não existir qualquer estudo que tenha estabelecido valores de corte para as contagens do CSA colocado no punho para as diferentes categorias de intensidade. Tão pouco existem na literatura equações para estimar o dispêndio energético através das contagens do CSA neste escalão etário.

Os principais resultados do presente estudo referem-se às diferenças existentes entre a intensidade de actividade física nos dias de semana e o fim de semana, à forte variabilidade intraindividual ao longo dos dias e à inexistência de diferenças entre os rapazes e as raparigas.

Contrariamente ao que seria de esperar, a maior intensidade de actividade física ocorreu, tanto nos rapazes como nas raparigas, durante os dias escolares, ocorrendo nos dias de fim de semana, particularmente no domingo, um decréscimo acentuado de actividade física. De facto, o fim de semana é, por excelência, um período de tempo livre, com inexistência de tarefas e deveres escolares, ao contrário dos dias de semana, onde existe a obrigatoriedade de permanecer na escola, pelo que o fim de semana deveria ser caracterizado por uma maior intensidade de actividade física, o que não aconteceu. Estes resultados não são confirmados pela literatura. $\mathrm{Na}$ realidade existe alguma contradição entre os resultados da presente investigação e outros estudos. Por exemplo, Trost et al. (29) encontraram, em crianças de 6 a 8 anos de idade, maior intensidade de actividade física ao fim de semana do que durante a semana, tanto em rapazes como em raparigas.

Estes resultados podem ficar a dever-se à fraca participação dos pais das crianças da amostra em actividades físicas e desportivas, o que parece ser uma realidade nacional. De facto, num estudo recente realizado nos Açores, Maia, Lopes e Morais (19) encontraram índices de participação muito baixos: $9 \%$ das mães e $25 \%$ dos pais. Também Mariovet (21) refere para a população portuguesa um índice de participação baixo - 23\%. A família é o meio social por excelência para a formação de hábitos de actividade física, e o fim de semana o período semanal mais adequado para a convivência familiar, dado que é durante este período que a criança permanece, em princípio, mais tempo e com maior proximidade com os pais. Várias investigações, tanto realizadas noutros países como em Portugal, referem níveis relativamente elevados de agregação familiar na actividade física, Por exemplo, Freedson e Evenson indicam um valor de $67 \%$ de agregação pai/filho e de $73 \%$ mãe/filho em crianças de 5 a 9 anos de idade. Em Portugal, Sá (24) verificou um valor entre $45 \%$ e $52 \%$ para a agregação pai/filho e entre $43 \%$ e 53 para a agregação mãe/filho em adolescentes.

Provavelmente as crianças da amostra permanecem mais tempo em casa durante o fim de semana, ocupadas com actividades de menor actividade, como por exemplo vendo televisão. A referência a uma vida fisicamente activa passa, também, pelo papel social activo, comprometido e actuante dos progenitores relativamente aos seus filhos. Se os filhos também aprendem pela imitação e referência à família, a promoção da actividade física nas crianças deve também passar pela promoção da actividade física regular entre os pais.

De acordo com o que era esperado, as crianças, sobretudo as do sexo masculino, apresentam uma grande variabilidade intraindividual na actividade física ao longo dos dias de observação. Ao longo dos quatro dias de observação o coeficiente $\mathrm{R}$ é de 0,56 para os rapazes e 0,76 para as raparigas. $\mathrm{O}$ valor do 
rapazes é mais baixo do que os encontrados por Trost et al. (29) em quatro dias de registo $(\mathrm{R}=0,77)$ e por Janz, et al. (17) $(\mathrm{R}=0,75)$. Nos dias de semana, nos rapazes, foi anotada uma maior variabilidade do que nas raparigas, tendo estas um valor de $\mathrm{R}$ bastante elevado $(0,90)$, o que indica fraca variabilidade ou, por outras palavras, consistência ou estabilidade na actividade física habitual. No fim de semana, tanto nos rapazes como nas raparigas, foi registada elevada variabilidade intraindividual, tendo as raparigas um valor de R muito próximo de $0(0,04)$. Janz, et al.

(17) encontraram, para um período de dois dias, um valor de $\mathrm{R}=0,59$. Os autores não indicam, no entanto, se os dias são dias de semana ou de fim semana. Ao longo dos dias da semana o período do intervalo/recreio escolar corresponde ao período de maior intensidade de actividade física. Este resultado é consistente com a literatura. Sleap e Warbuston (27), num estudo com crianças de 4 regiões de Inglaterra, verificaram que estas eram mais activas durante os intervalos lectivos do que durante o tempo livre passado fora da escola.

A elevada variabilidade intraindividual verificada é consentânea com as características da actividade física das crianças deste intervalo etário. De facto, a actividade física das crianças caracteriza-se por períodos curtos de actividade física intensa, intercalados com períodos frequentes de inactividade ou actividade física menos intensa $(32,4)$.

Ao longo de cada dia de observação o perfil de actividade física dos rapazes e das raparigas é idêntico, não havendo diferenças significativas entre os sexos, quer ao longo de cada dia, quer entre cada dia de observação. Estes resultados não são confirmados nem infirmados pela literatura; na realidade, os resultados das investigações são contraditórios. Welsman e Armstrong (33) verificaram, através da monitorização da frequência cardíaca, que os rapazes passavam significativamente mais tempo em actividades físicas moderadas e intensas do que as raparigas. No entanto, Atkins et al. (3) não encontram diferenças significativas entre rapazes e raparigas. Por outro lado, Santos (25) ao analisar a actividade habitual em 157 indivíduos de ambos os sexos com idades compreendidas entre os 8 e os 16 anos, através do monitor de actividade física CSA, apurou que os rapazes foram mais activos que as raparigas.
Manios, Kafatos e Markakis (20) encontraram, através de questionário, tempos superiores de actividade física para os rapazes, embora a diferença não seja significativa. Trost et al. (30) verificaram que os rapazes apresentavam significativamente maior participação em actividades físicas moderadas do que as raparigas, mas encontraram diferenças significativas entre os sexos na participação em actividades físicas intensas.

Em conclusão, não foram encontradas diferenças significativas entre a intensidade de actividade física registada nos rapazes e nas raparigas. As crianças de ambos os sexos apresentaram uma forte variabilidade intraindividual na actividade física habitual, sobretudo ao fim de semana. Nos dias de semana as crianças apresentaram uma maior intensidade de actividade física do que nos dias de fim de semana, sendo o Domingo o dia que apresenta uma menor intensidade de actividade física. Durante os dias de semana, foi no período do intervalo/recreio escolar que foi registada maior intensidade de actividade física.

\section{CORRESPONDÊNCIA}

Vítor Pires Lopes

Escola Superior de Educação de Bragança

Campus de Santa Apolónia

Apartado 1101

5301-856 Bragança

Portugal

vplopes@ipb.pt 


\section{BIBLIOGRAFIA}

1. American College of Sports Medicine (1988). Opinion statement on physical fitness in children and youth. Med. Sci. Sports Exerc. 20: 422-423.

2. American College of Sports Medicine (1991). Guidelines for exercise testing and prescriptions ( $4^{\mathrm{a}} \mathrm{ed}$.). Filadélfia: Lea and Febiger.

3. Atkins, S.; Stratton, G.; Dugdill, L.; Reilly, T. (1997). The free-living physical activity of schoolchildren: a longitudinal study. In: N. Armstrong; B. J. Kirby; J. R. Welsman (eds.) Children and Exercise XIX. Promoting Health and Well-Being. Londres: E \& Spon.

4. Baley, R. C.; Olson, J.; Pepper, S. L.; Porszaz, J.; Barstow, T. L.; Cooper, D. M. (1995). The level and tempo of children's physical activity. An observation study. Med. Sic. Sports Exerc. 27: 1033-1041.

5. Baranowski, T.; Moor, C. (2000). How many days was that? Intra-individual variability and physical activity assessment. Res Q. Exerc. Sport. 71(2): 74-78.

6. Bar-Or, O. (1987). A Commentary to Children and Fitness: a public health perspective. Res. Q. Exerc. Sport. 58 (4): 304-307. 7. Blair, S. N. (1993). 1993 C. H. McCloy Research Lecture: physical activity, physical fitness, and health. Res. Q. Exerc. Sport. 64 (4): 365-376.

8. Blair, S. N.; Clark, D. G.; Cureton, K. J.; Powell, K. E. (1989). Exercise and fitness in childhood: implications for lifetime of health. In: C. V. Gisolfi; D. R. Lamb (eds.) Perspectives in Exercise Science and Sport Medicine; Vol 2; Youth, Exercise, and Sport. Indianapolis: Benchmark Press.

9. Blair, S. N.; Kohl, H. W.; Paffenbarger, R. S.; Clark, D. G.; Cooper, K. H.; Gibbons, L. W. (1989). Physical fitness and allcause mortality: a prospespective study of healthy men and women. JAMA. 262: 2395-2401.

10. Clark, D. G.; Blair, S. N. (1988). Physical activity and prevention of obesity in childhood. In: N. A. Krasneger, G. D. Grave; N. Kretchmer (eds.) Childhood Obesity: a biobehavioral perspective. Caldwell, N.J: Telford Press.

11. Corbin, C. B. (1987). Youth Fitness, Exercise and Health: there is much to be done. Res. Q. Exerc. Sport. 58 (4): 308-314. 12. Council for Physical Education for Children (1998). Physical activity for children: a statement of guidelines. Reston, VA: NASPE Publication.

13. Durant, R. H.; Linder, C. W.; Mahoney, O. M. (1983). The relationship between habitual physical activity and serum lipoproteins in white male adolescents. Journal of Adolescent Health Care. 4: 235-239.

14. Fletcher, G. F.; Blair, S. N.; Blumenthal, J.; Caspersen, C.; Chaitman, B.; Epstein, S.; Falls, H.; Froelicher, E. S. S.; Pina, I. L. (1992). Statement on exercise: benefits and recommendations for physical activity programs for all americans. A statement for health professionals by the Committee on Exercise and Cardiac Rehabilitation of the Council on Clinical Cardiology, American Heart Association. Circulation. 86: 340344.

15. Freedson, P. S.; Evenson, S. (1991). Familial aggregation in physical activity. Res. Q. Exerc. Sport. 62 (4): 384-389. 16. Freedson, P. S.; Melanson, E.; Sirard, J. (1998). Calibration of the Computer Science and Applications, Inc. accelerometer. Med. Sci. Sports Exerc. 30 (5): 777-781.

17. Janz, K. F.; Witt, J.; Mahoney, L. T. (1995) The stability of children's physical activity as measured by accelerometry and self-report. Med. Sci. Sports Exerc. 27(9): 1326-1332.
18. Magalhães, M. L. R. (2001). Padrão de actividade física. Estudo em crianças de ambos os sexos do $4^{\circ}$ ano de escolaridade. Dissertação de mestrado. Faculdade de Ciências do Desporto e de Educação Física. Universidade do Porto. Porto.

19. Maia, J. A. R.; Lopes, V. P.; Morais, A. F. P. (2001). Actividade física e aptidão física associada à saúde. Um estudo de epidemiologia genética em gémeos e suas famílias do arquipélago dos Açores. Porto: Faculdade de Ciências do Desporto e Educação Física e Direcção Regional de Educação Física e Desporto dos Açores.

20. Manios, Y.; Kafatos, A.; Markakis, G. (1998). Physical activity of 6-year-old children: validation o two proxy reports. Pedia. Exerc. Sci. 10: 176-188.

21. Mariovet, S. (2001). Hábitos desportivos da população portuguesa. Lisboa: Instituto Nacional de Formação e Estudos do Desporto.

22. Melanson, E.; Freedson, P. S. (1995). Validity of the computer science and applications, Inc. activity monitor. Med. Sci. Sports Exerc. 27(8): 934-940.

23. Riople, D. A.; Boerth, R. C.; Coates, T. J.; Hennekens, C. H.; Miller, W. M.; Weidman, W. H. (1986) Coronary risk factor modification in children: exercise. A statement for physicians by Committee on Atherosclerosis and Hypertension in Childhood of the Council on Cardiovascular Disease in Young. Circulation 74: 1189A-1191A.

24. Sá, M. P. C. M. (2000). Transmissibilidade nos hábitos de actividade física. Um estudo em alunos do sexo masculino do $10^{\circ}$ ao $12^{\circ}$ ano de escolaridade. Dissertação de mestrado. Faculdade de Ciências do Desporto e Educação Física. Universidade do Porto.

25. Santos, M. P. M. (2000). Avaliação da actividade física habitual em crianças e adolescentes do grande Porto. Dissertação de

Mestrado. Faculdade de Ciências do Desporto e de Educação Física. Universidade do Porto. Porto.

26. Simons-Morton, B.G.; Baranowski, T.; O'Hara, N.; Parcel, G. S.; Huang, I.W.; Wilson, B. (1990). Children's frequency of participation in moderate to vigorous physical activities. Res. $Q$. Exerc. Sport. 61 (4): 307-314.

27. Sleap, M.; Warbuston, P. (1992). Physical activity levels of 5-11-years-old children in England as determined by continuous observation. Res. Q. Exerc. Sport. 63 (3): 238-245. 28. Swartz, A. M.; Strath, S. J.; Bassett, D. R.; O’Brien, W. L.; King, G. A.; Ainsworth, B. E. (2000). Estimation of energy expenditure using CSA accelerometers at hip and wrist sites. Med. Sci. Sports Exerc. 32 (9 Supplement): S450-S456.

29. Trost, S. G.; Pate, R. R.; Freedson, P. S.; Sallis, J. F.; Taylor, W. C. (2000). Using objective physical activity measures with youth: how many days of monitoring are needed? Med. Sci. Sports Exerc. 32 (2): 426-431.

30. Trost, S. G.; Pate, R. R.; Ward, D. S.; Sauders, R.; Riner, W. (1999). Correlates of objectively measured physical activity in preadolescent youth. Am. J. Prev. Med. 17 (2): 120-126.

31. U.S Department of Health and Human Services. (1991). Healthy People 2000: national Health Promotion and Disease Prevention Objectives. Washington. U.S. Government Printing Office.

32. Welk, G. J.; Corbin, C. B.; Dale, D. (2000). Measurement issues in the assessment of physical activity in children. Res. $Q$. Exerc. Sport. 71(29): 59-73.

33. Welsman, J. R.; Armstrong, N. (1997). Physical activity patterns of 5 to 11 year old children. In: N. Armstrong; B. J. Kirby; J. R. Welsman (eds.) Children and Exercise XIX. Promoting Health and Well-Being. Londres: E \& Spon. 